\title{
Cisplatin and Etoposide with Concurrent Radiotherapy in Locally Advanced Thymic Carcinoma
}

\author{
(1) Gonca HANEDAN USLU,' (1) Çağlar YILDIZ AKDENIZ, ${ }^{2}$ (i) Nihat YEŞILIRMAK ${ }^{3}$ \\ 'Department of Radiation Oncology, Kanuni Training and Research Hospital, Trabzon-Turkey \\ ${ }^{2}$ Department of Medical Pathology, Kanuni Training and Research Hospital, Trabzon-Turkey \\ ${ }^{3}$ Department of Thoracic Surgery, Kanuni Training and Research Hospital, Trabzon-Turkey
}

\section{Dear Editor,}

Thymoma is the most common type of thymic tumors, whereas thymic carcinomas are very rare tumors with poor prognosis and an incidence of $0.05 \%$ and were first reported by Shimosato et al.[1] in 1977. Surgery is the mainstay of therapy in resectable cases. Multimodal approach plays a crucial role in partially resectable or nonresectable cases. However, due to the rare nature of these tumors, there have been no extensive studies on pre- and post-operative chemoradiotherapy and dose selection for chemotherapy regimens and radiotherapy. Here, we describe the case of a patient with partially resectable locally advanced thymic carcinoma in whom $40 \%$ reduction was achieved after concurrent chemoradiotherapy.

A 35-year-old male patient presented to the clinic with chest pain that had persisted for 1 month. Posteroanterior chest radiography result revealed a mass in the middle mediastinum. Pre-operative magnetic resonance imaging (MRI) results revealed the soft-tissue density mass lesion, with a few millimetric calcification foci (approximately $84 \times 75 \mathrm{~mm}$ ), adjacent to the left upper lobe paramediastinal area in the anterior mediastinum (Fig. 1). Partial resection of the mediastinal mass was performed with the left thoracotomy. On gross pathological examination, the tumor was a $3 \mathrm{~cm}$ diameter, non-encapsulated solid mass lacking broad fibrous septae on the cut surface. Surgical margin was positive because of incomplete resection due to proximity to the big vessels. Microscopically, the tumor was composed of sheets of cohesive non-keratinizing squamous cells displaying invasion to the lungs. Immunohistochemical studies support the diagnosis of thymic carcinoma with positive CD5, CD117, CK5/6, and P63

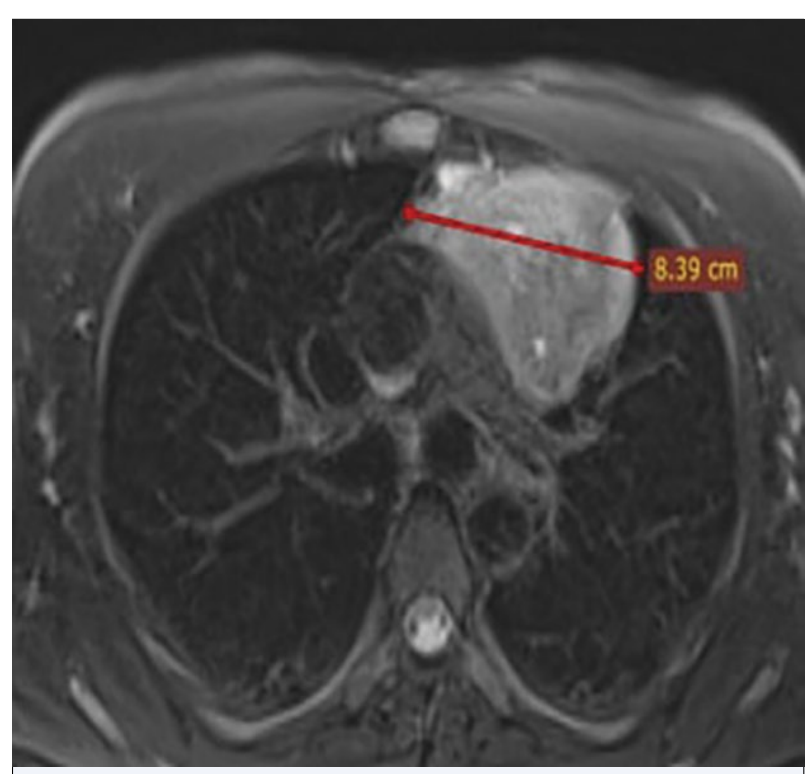

Fig. 1. Pre operative magnetic resonance imaging.

staining. Positive CD5 and CD117 indicate a thymic origin. Negative TTF-1, CK7, and CK20 ruled out metastasis. No lymphovascular or perineural invasion was observed (Fig. 2a-d). The Masaoka stage was identified as IVa. There was no evidence to indicate myasthenia gravis. Following partial resection, chemoradiotherapy was administered. Cisplatin $50 \mathrm{mg} / \mathrm{m}^{2}$ on days $1,8,29$, and 36 ; etoposide $50 \mathrm{mg} / \mathrm{m}^{2}$ on days $1-5$ and 29-33; and radiotherapy at a total dose of 59.4 Gy at $1.8 \mathrm{~Gy} /$ fraction helical tomotherapy for 33 days were planned. When the mass shrank at $39.6 \mathrm{~Gy}$, adaptive planning was applied, and the radiotherapy doses administered to the lung and heart were reduced. Chest 

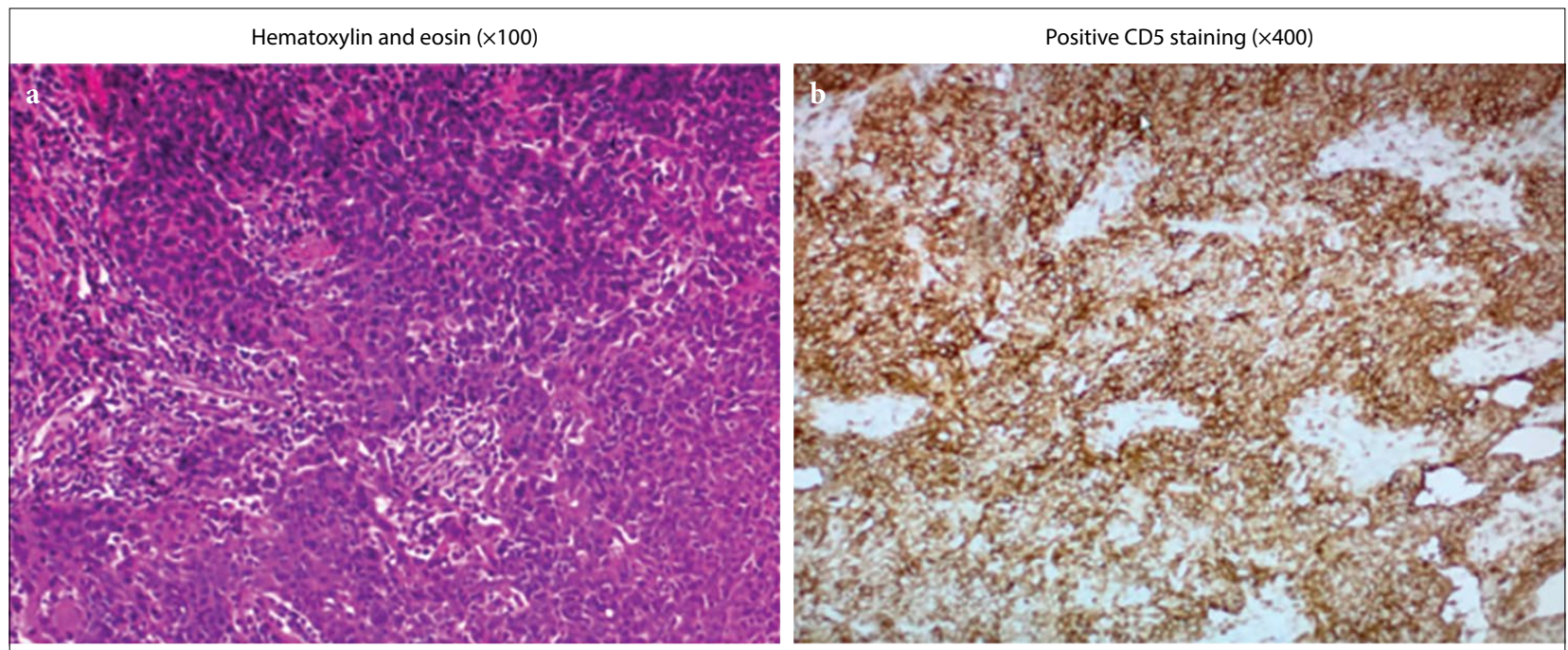

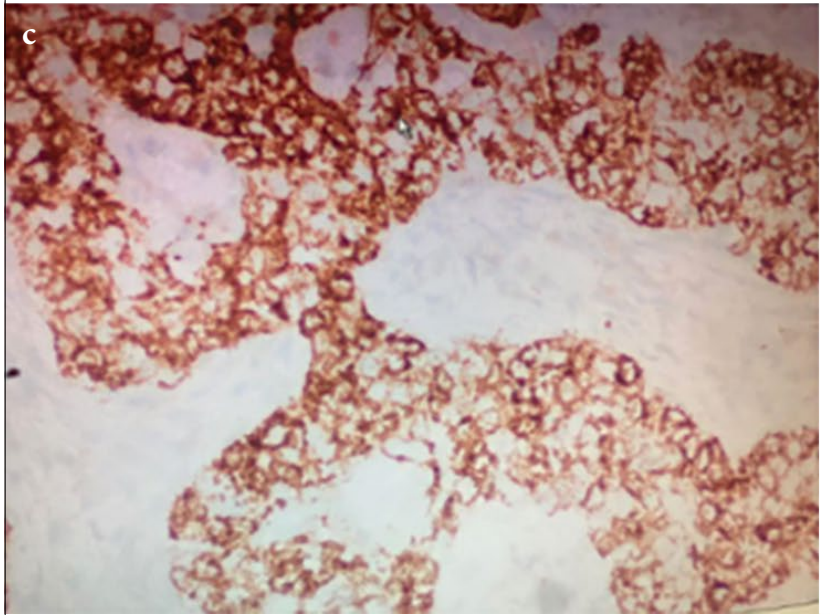

Positive CK5/6 staining $(\times 400)$

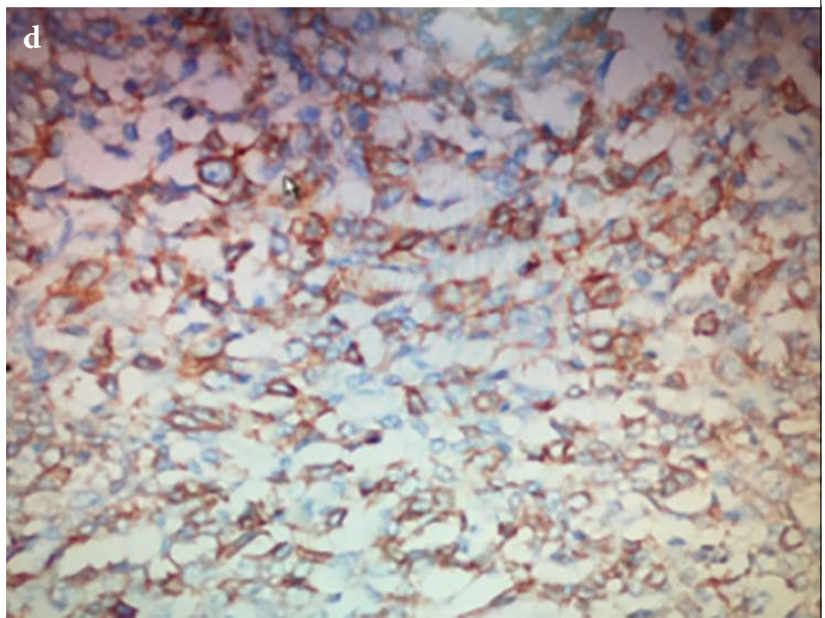

Positive CD117 staining $(\times 400)$

Fig. 2. (a) Sheets of non-keratinising malignant squamous cells with infiltrative growth pattern (hematoxylin and eosin $\times 100)$, (b) Positive CD5 staining (×400), (c) Positive CK5/6 (×400), (d) Positive CD117 staining $(\times 400)$.

MRI performed 2 months after the chemoradiotherapy revealed that the mass had shrunk by $40 \%$ (Fig. 3 ). The patient is stable at the $6^{\text {th }}$ year follow-up.

Thymic carcinoma is a rare aggressive neoplasm. It is usually diagnosed at the advanced stage of III or IV according to the Masaoka staging system. Although complete surgical resection is the main treatment modality, it is not always feasible due to invasion of the neighboring vessels, lung tissues, pericardium, and pleura. Alternatively, several treatment modalities, such as surgery following induction chemotherapy and surgery following chemoradiotherapy, are available. There is no standard treatment due to the uncommon nature of the disease. Some studies have shed light on the use of induction chemotherapy regimen.[2-4] In a study that reported an overall survival rate of $78 \%$ with surgery following induction chemotherapy followed by radiotherapy, the neoadjuvant chemotherapy setting for eight patients consisted of cisplatin $\left(75 \mathrm{mg} / \mathrm{m}^{2}\right.$ on day $1)$, epirubicin hydrochloride $\left(100 \mathrm{mg} / \mathrm{m}^{2}\right.$ on day 1$)$, and etoposide $\left(120 \mathrm{mg} / \mathrm{m}^{2}\right.$ on days 1,3 , and 5) repeated 3 times every 3 weeks; the induction chemotherapy regimen for the remaining patients consisted of cisplatin $\left(50 \mathrm{mg} / \mathrm{m}^{2}\right)$, adriblastin $\left(50 \mathrm{mg} / \mathrm{m}^{2}\right)$, and cyclophosphamide $\left(500 \mathrm{mg} / \mathrm{m}^{2}\right)$ repeated 3 times every 3 weeks. A dose of 40 Gy was usually administered to patients undergoing complete resection and 50-60 Gy to those undergoing partial resection; the mediastinum or residual tumor areas were irradiated in 3-5 weeks with five fractions per week.[2] In a majority of reported cases, cisplatin is a key agent for chemotherapy against thymic tumors and was included in all of the reported regimens: 


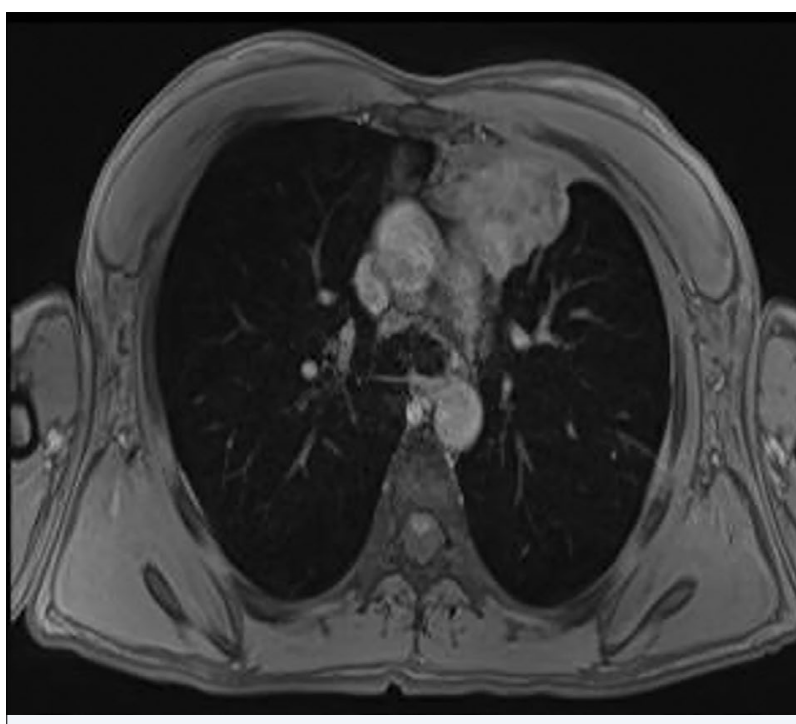

Fig. 3. After chemoradiotherapy magnetic resonance imaging.

Adriamycin/cisplatin/vincristine/cyclophosphamide or cisplatin/etoposide,[3] cisplatin/epirubicin/etoposide or cisplatin/adriamycin/cyclophosphamide, and adriamycin/cisplatin/cyclophosphamide/prednisone.[4] Theoretically, induction chemotherapy with concurrent radiotherapy seems to be ideal for these cases similar to that for other malignancies; however, limited prospective or retrospective studies and case reports are available. Therefore, we selected cisplatin $50 \mathrm{mg} / \mathrm{m}^{2}$ on days $1,8,29$, and 36 and etoposide $50 \mathrm{mg} / \mathrm{m}^{2}$ on days $1-5$ and 29-33 with concurrent radiotherapy for induction treatment, although the supporting evidence is currently limited. In our case, as only partial resection was possible due to vascular invasion, concurrent chemoradiotherapy was actually planned for induction purposes. Therefore, the initial planned dose of radiotherapy was $59 \mathrm{~Gy}$. We reassessed the possibility of surgery with chest MRI at $50.4 \mathrm{~Gy}$ (day 28 of radiotherapy). Since it was considered unresectable based on the decision of the chest surgery clinic, we completed radiotherapy at a dose of 59 Gy concurrently with chemotherapy. Thus, we demonstrated the efficacy of chemoradiotherapy with the use of cisplatin and etoposide in our case.

Paclitaxel is another preferred induction chemotherapy agent. It is a new agent that induces excessive polymerization of tubulin and has demonstrated clinical activity in a wide variety of malignancies, including ovarian, breast, head-and-neck, and lung cancers. Morio et al.[5] reported a case of Stage IVb thymic carcinoma with lymph node metastasis and achieved complete resection after induction therapy with weekly paclitaxel plus cisplatin and concurrent radiotherapy (total dose $40 \mathrm{~Gy}$ ). Another case of advanced thymic carcinoma treated with induction docetaxel, which is also a new agent classified as a taxan-like paclitaxel, plus cisplatin, and concurrent radiotherapy (total dose $40 \mathrm{~Gy}$ ) also achieved complete resection. [6]

Although systemic workups did not detect any distant metastases, the patient was diagnosed as Stage IVb according to the Masaoka staging system[7] because of mediastinal lymph node metastasis. Two cycles of chemotherapy consisting of paclitaxel $\left(180 \mathrm{mg} / \mathrm{m}^{2}, 3 \mathrm{~h}\right)$ and cisplatin $\left(80 \mathrm{mg} / \mathrm{m}^{2}, 1 \mathrm{~h}\right)$ on day 1 were administered every 3 weeks.

Anthracycline-based regimens are the current standard of care for thymic carcinomas according to the results of various Phase II clinical trials, but they have not been tested in a Phase III study. Fornasiero et al.[8] reported their 13 years of experience in treating $37 \mathrm{pa}-$ tients with Stages III and IV thymic carcinoma with cisplatin, doxorubicin, vincristine, and cyclophosphamide combination. The ORR was $91.8 \%$; with $43 \%$ achieving $\mathrm{CR}$, but the median survival time was only 15 months. Loehrer et al.[9] reported a $50 \%$ ORR with $10 \%$ of patients achieving CR and a median survival patient with thymic carcinoma with metastatic or locally progressive recurrent disease were treated with paclitaxel. There is another Phase II study using a multidisciplinary approach with induction chemotherapy followed by surgical resection, radiation therapy, and consolidation chemotherapy for unresectable thymoma. In this Phase II study; 22 patients received induction chemotherapy with PAC plus prednisone for three cycles. The authors reported that induction chemotherapy resulted in a $14 \%$ CR and a 63\% PR rate. However, anthracyclines are known to be associated with cardiomyopathy, especially when combined with radiotherapy. Therefore, non-anthracycline regimens may be preferable for patients treated with chemoradiotherapy.[4]

The European Organization for Research and Treatment of Cancer conducted a study in which 16 patients with advanced or recurrent thymoma were treated with cisplatin and etoposide. In this trial, five patients achieved CR and four achieved PR (ORR, 56\%).[10] On the basis of a single agent activity of ifosfamide in thymoma, 20 patients with advanced thymoma and eight patients with thymic carcinoma were treated with etoposide, ifosfamide, and cisplatin in an intergroup trial conducted by ECOG. An ORR of 35\% and 25\% was reported in patients with thymoma and thymic carcinoma, respectively.[11] Grassin et al.[12] reported similar poor results (PR, 25\%) in a study of 16 patients treated with etoposide, ifosfamide, and cisplatin. These cisplatin plus etoposide-based regimens produced apparently inferior response rates to those previously 
reported for anthracycline-based regimens.[13] As anthracycline-based chemotherapy can cause cardiac toxicities and cardiomyopathy, etoposide plus cisplatin is a potential alternative if radiotherapy is also to be administered. Furthermore, etoposide plus cisplatin combined with radiotherapy is the standard treatment for patients with localized advanced non-small-cell lung cancer[14,15] and local small-cell lung cancer[16] and has generally been well-tolerated by such patients.

In summary, combined cisplatin plus etoposide for thymic carcinoma is effective and reasonably well-tolerated, particularly with concurrent radiotherapy. Despite the limited evidence to support the use of cisplatin plus etoposide as a treatment for advanced thymic carcinoma, it is an alternative chemotherapy when anthracycline-based regimens cannot be used. Given the rarity of this tumor, prospective randomized trials are unlikely. Cumulative data, however, suggest that anthracyclinebased regimens should remain the standard of care for these patients. However, for patients who can be treated with concurrent radiotherapy, etoposide plus cisplatin is a potential chemotherapy regimen for this malignancy.

\section{References}

1. Shimosato Y, Kameya T, Nagai K, Suemasu K. Squamous cell carcinoma of the thymus. An analysis of eight cases. Am J Surg Pathol 1977;1(2):109-21.

2. Venuta F, Rendina EA, Longo F, De Giacomo T, Anile $\mathrm{M}$, Mercadante E, et al. Long-term outcome after multimodality treatment for stage III thymic tumors. Ann Thorac Surg 2003;76(6):1866-72; discussion 1872.

3. Bretti S, Berruti A, Loddo C, Sperone P, Casadio C, Tessa M, et al; Piemonte Oncology Network. Multimodal management of stages III-IVa malignant thymoma. Lung Cancer 2004;44(1):69-77.

4. Kim ES, Putnam JB, Komaki R, Walsh GL, Ro JY, Shin HJ, et al. Phase II study of a multidisciplinary approach with induction chemotherapy, followed by surgical resection, radiation therapy, and consolidation chemotherapy for unresectable malignant thymomas: final report. Lung Cancer 2004;44(3):369-79.

5. Morio A, Nakahara K, Ohse Y, Tahara M, Goto T, Yakumaru K et al. Efficacy of induction chemoradiotherapy in thymic cancer: report of a successful case and review of the literature. Int J Clin Oncol 2002;7:201-4.

6. Shintani Y, Ohta M, Hazama K, Miyoshi S, Kagisaki $\mathrm{K}$ and Matsuda H: Thymic carcinoma successfully resected with superior vena cava after chemoradiotherapy. Jpn J Thorac Cardiovasc Surg 2001;49:717-21.

7. Masaoka A, Monden Y, Nakahara K, Tanioka T. Follow-up study of thymomas with special reference to their clinical stages. Cancer 1981;48(11):2485-92.

8. Fornasiero A, Daniele O, Ghiotto C, Piazza M, Fiore-Donati L, Calabró F, et al. Chemotherapy for invasive thymoma. A 13-year experience. Cancer 1991;68(1):30-3.

9. Loehrer PJ Sr, Kim K, Aisner SC, Livingston R, Einhorn LH, Johnson D, et al. Cisplatin plus doxorubicin plus cyclophosphamide in metastatic or recurrent thymoma: final results of an intergroup trial. The Eastern Cooperative Oncology Group, Southwest Oncology Group, and Southeastern Cancer Study Group. J Clin Oncol 1994;12(6):1164-8.

10. Giaccone G, Ardizzoni A, Kirkpatrick A, Clerico M, Sahmoud T, van Zandwijk N. Cisplatin and etoposide combination chemotherapy for locally advanced or metastatic thymoma. A phase II study of the European Organization for Research and Treatment of Cancer Lung Cancer Cooperative Group. J Clin Oncol 1996;14(3):814-20.

11. Highley MS, Underhill CR, Parnis FX, Karapetis C, Rankin E, Dussek J, et al. Treatment of invasive thymoma with single-agent ifosfamide. J Clin Oncol 1999;17(9):2737-44.

12. Grassin F, Paleiron N, André M, Caliandro R, Bretel JJ, Terrier P, et al. Combined etoposide, ifosfamide, and cisplatin in the treatment of patients with advanced thymoma and thymic carcinoma. A French experience. J Thorac Oncol 2010;5(6):893-7.

13. Loehrer PJ Sr, Jiroutek M, Aisner S, Aisner J, Green M, Thomas CR Jr, et al. Combined etoposide, ifosfamide, and cisplatin in the treatment of patients with advanced thymoma and thymic carcinoma: an intergroup trial. Cancer 2001;91(11):2010-5.

14. Albain KS, Swann RS, Rusch VW, Turrisi AT ${ }^{\text {rd }}$, Shepherd FA, Smith C, et al. Radiotherapy plus chemotherapy with or without surgical resection for stage III non-small-cell lung cancer: a phase III randomised controlled trial. Lancet 2009;374(9687):379-86.

15. Hanna N, Neubauer M, Yiannoutsos C, McGarry R, Arseneau J, Ansari R, et al; Hoosier Oncology Group; US Oncology. Phase III study of cisplatin, etoposide, and concurrent chest radiation with or without consolidation docetaxel in patients with inoperable stage III non-small-cell lung cancer: the Hoosier Oncology Group and U.S. Oncology. J Clin Oncol 2008;26(35):5755-60.

16. Takada M, Fukuoka M, Kawahara M, Sugiura T, Yokoyama A, Yokota S, et al. Phase III study of concurrent versus sequential thoracic radiotherapy in combination with cisplatin and etoposide for limited-stage small-cell lung cancer: results of the Japan Clinical Oncology Group Study 9104. J Clin Oncol 2002;20(14):3054-60. 